\title{
Emergent Anyon Distribution in the Unruh Effect
}

\author{
Satoshi Ohya \\ Institute of Quantum Science, Nihon University \\ Kanda-Surugadai 1-8-14, Chiyoda, Tokyo 101-8308, Japan \\ ohya@phys.cst.nihon-u.ac.jp
}

(Dated: November 8, 2017)

\begin{abstract}
We point out that, when the Unruh-DeWitt detector couples to a scalar primary operator of $d$-dimensional conformal field theory, the detector's power spectrum generally obeys the thermal distribution for $(1+1)$-dimensional anyons.
\end{abstract}

\section{Introduction}

A uniformly accelerating observer of constant proper acceleration $a(>0)$ sees the vacuum for an inertial observer as a thermal state of temperature $T=a /(2 \pi)$. This is the Unruh effect [1] and has been vastly studied over the last forty years both from physical and mathematical perspectives. From the physical viewpoint, the Unruh effect provides a theoretical laboratory for understanding quantum phenomena in gravitational field background, whose typical example is the Hawking radiation of black hole [2]. It also provides a nice pedagogical example of Takahashi-Umezawa thermofield dynamics [3] -an interplay between entanglement and thermalization. From the mathematical viewpoint, on the other hand, the Unruh effect provides a nice geometrical realization of TomitaTakesaki modular theory of operator algebras, in which the modular operator and modular conjugation are simply given by the complexified Lorentz boost and CPT conjugate (with partial reflection), respectively. It also provides the interplay between the Bisognano-Wichmann theorem [4,5] and the Kubo-Martin-Schwinger (KMS) thermal equilibrium condition [6], based on which the Unruh effect can be proved mathematically. Though it is shown axiomatically by Sewell [7] that correlation functions with respect to the inertial vacuum satisfy the KMS conditions with respect to the Lorentz boost, whereby the thermality of inertial vacuum for accelerating observers is rigorously proved, there still remain puzzles in the Unruh effect. Among them is the Takagi's statistics inversion [8], which is an apparent phenomenon that interchanges the Bose-Einstein and Fermi-Dirac distributions. In the mid-1980s Takagi studied the power spectrum of the Unruh-DeWitt detector [9] coupled to a massless scalar field in generic spacetime dimension $d$ and found that, when $d$ is even, the detector's power spectrum obeys the Bose-Einstein distribution. This is as naively expected since the power spectrum is simply given by the Fourier transform of scalar two-point Wightman function. However, when $d$ is odd, he found that the power spectrum obeys the Fermi-Dirac distribution even without any fermionic degrees of freedom. Soon after this discovery, Ooguri pointed out [10] that this puzzle can be explained in terms of the absence of Huygens principle in odd dimensional spacetimes [11]. In other words, this apparent phenomena can be understood from the analytic structure of two-point Wightman functions and resulting anti-commutativity for time-like separated scalar correlators, which can happen only for massless non-interacting fields in odd dimensions. Instead of massless free fields, in this short note we shall consider a primary operator of conformal field theory, for which the Huygens principle is violated as well. The goal of this note is to show that, when the Unruh-DeWitt detector couples to a scalar primary operator of conformal field theory with non-vanishing anomalous dimension, the detector's power spectrum generally obeys the 
thermal distribution for $(1+1)$-dimensional anyons that is derived by Liguori, Mintchev, and Pilo in 1999 by making use of the bosonization technique [12]. We shall see that the Takagi's statistics inversion is then generalized to a statistics interpolation-an apparent phenomenon which interpolates the Bose-Einstein and Fermi-Dirac distributions in a continuous way. ${ }^{1}$ The scaling dimension of primary operator turns out to play the role of the so-called statistical parameter. Before embarking on this statistics interpolation, however, let us first briefly recall the Takagi's findings.

\section{Statistics Inversion: A Review}

The central objects of statistics inversion are the so-called Unruh-DeWitt detector introduced by DeWitt [9] and its power spectrum (also known as the response function). Let us consider a uniformly accelerating point-like detector in the $d$-dimensional Minkowski spacetime. Let us further suppose that the whole spacetime is filled with a quantum field theory. The combined system of detector and quantum field theory is then schematically described by the action

$$
S_{\text {total }}=S_{\text {detector }}+S_{\mathrm{QFT}}+S_{\text {int }}
$$

where $S_{\text {detector }}$ and $S_{\mathrm{QFT}}$ are the actions of detector and background quantum field theory whose explicit forms are not necessary in the following discussions. For simplicity we shall focus on a hermitian scalar field as the background. DeWitt considered that the detector has a "monopole moment" $m(\tau)$ and interacts with the background scalar field $\phi(x)$ via the following term:

$$
S_{\text {int }}=\int_{-\infty}^{\infty} d \tau m(\tau) \phi(x(\tau)),
$$

where $\tau$ is the detector's proper time and $x^{\mu}(\tau)$ is the worldline for the uniformly accelerating detector given by

$$
x^{0}(\tau)=\frac{1}{a} \sinh (a \tau), \quad x^{1}(\tau)=\frac{1}{a} \cosh (a \tau), \quad x_{\perp}(\tau)=\text { constant }
$$

We wish to know the transition amplitude for the process $\left|E_{i}\right\rangle \otimes|0\rangle \rightarrow\left|E_{f}\right\rangle \otimes|\Phi\rangle$, where $\left|E_{i / f}\right\rangle \in$ $\mathcal{H}_{\text {detector }}$ is the initial (final) state of the detector and $|0\rangle \in \mathcal{H}_{\mathrm{QFT}}$ is the vacuum for inertial observers. The final state $|\Phi\rangle \in \mathcal{H}_{\mathrm{QFT}}$ is an arbitrary basis of background quantum field theory which is assumed to satisfy the completeness $\sum_{\Phi}|\Phi\rangle\langle\Phi|=1$. In the interaction picture, the transition amplitude is given by the matrix element $A\left(E_{f}, \Phi \mid E_{i}, 0\right)=\left\langle E_{f}, \Phi\left|\left(T \exp \left(i S_{\text {int }}\right)-1\right)\right| E_{i}, 0\right\rangle$ which, at the linear order of perturbation theory, takes the following form:

$$
A\left(E_{f}, \Phi \mid E_{i}, 0\right) \simeq i\left\langle E_{f}|m(0)| E_{i}\right\rangle \int_{-\infty}^{\infty} d \tau \mathrm{e}^{i\left(E_{f}-E_{i}\right) \tau}\langle\Phi|\phi(x(\tau))| 0\rangle .
$$

The transition probability per unit time-the transition rate-is then given by summing over all possible final states:

$$
R\left(E_{f} \mid E_{i}\right)=\frac{1}{T} \sum_{\Phi}\left|A\left(E_{f}, \Phi \mid E_{i}, 0\right)\right|^{2} \simeq\left|\left\langle E_{f}|m(0)| E_{i}\right\rangle\right|^{2} F(\omega),
$$

where $T=\int_{-\infty}^{\infty} d \tau$ is the total time for the process. Here $F(\omega)$ is the so-called detector's power spectrum and given by the Fourier transform of two-point Wightman function along the detector's worldline:

$$
F(\omega)=\int_{-\infty}^{\infty} d \tau \mathrm{e}^{-i \omega \tau}\langle 0|\phi(x(\tau)) \phi(x(0))| 0\rangle, \quad \omega=E_{f}-E_{i} .
$$

\footnotetext{
${ }^{1}$ Yet another statistics interpolation was discussed by Sriramkumar [13].
} 
Let us now focus on a massless free scalar field $\phi(x)$. In $d$-dimensional spacetime $\phi(x)$ has the mass dimension $(d-2) / 2$ and hence its two-point Wightman function $\langle 0|\phi(x) \phi(y)| 0\rangle$ is basically given by the power function $z^{-(d-2) / 2}$ of the Poincaré invariant $z=(x-y)^{2}=-\left(x^{0}-y^{0}\right)^{2}+|x-y|^{2}$. There is, however, a subtlety if $d$ is odd and $\phi(x)$ and $\phi(y)$ are time-like separated because in this case $z^{-(d-2) / 2}$ has the branch cut along the negative $z$-axis and hence its value depends on how one approaches the negative $z$-axis. A careful analysis shows that $z=(x-y)^{2}$ should be chosen as $z=|z| \mathrm{e}^{i \pi}$ for $(x-y)^{2}<0$ and $x^{0}-y^{0}>0$ and as $z=|z| \mathrm{e}^{-i \pi}$ for $(x-y)^{2}<0$ and $x^{0}-y^{0}<0 .^{2}$ Indeed, by canonically quantizing the field $\phi(x)$ one can see that the two-point Wightman function takes the following forms:

$$
\langle 0|\phi(x) \phi(y)| 0\rangle= \begin{cases}\frac{\Gamma\left(\frac{d-2}{2}\right)}{4 \pi^{\frac{d}{2}}} \frac{1}{\left[(x-y)^{2}\right]^{\frac{d-2}{2}}} & \text { for }(x-y)^{2}>0, \\ \frac{\Gamma\left(\frac{d-2}{2}\right)}{4 \pi^{\frac{d}{2}}} \frac{e^{-i \operatorname{sgn}\left(x^{0}-y^{0}\right) \frac{d-2}{2} \pi}}{\left[-(x-y)^{2}\right]^{\frac{d-2}{2}}} & \text { for } \quad(x-y)^{2}<0,\end{cases}
$$

where $\operatorname{sgn}(x)=x /|x|(\operatorname{sgn}(0)=0)$ is the sign function. The power spectrum (6) is evaluated by just substituting the worldline (3) into the Wightman function (7) and then Fourier transform with respect to the proper time. To do this, one should pay attention to the following two facts: the first is that any two points lying on the worldline (3) are always time-like separated; that is, $\left(x(\tau)-x\left(\tau^{\prime}\right)\right)^{2}=-\left(4 / a^{2}\right) \sinh ^{2}\left(a\left(\tau-\tau^{\prime}\right) / 2\right)<0$ for any $\tau-\tau^{\prime} \neq 0$. The second is that, since $x^{0}(\tau)=(1 / a) \sinh (a \tau)$ is a monotonically increasing function of $\tau$, the sign function $\operatorname{sgn}\left(x^{0}(\tau)-\right.$ $\left.x^{0}\left(\tau^{\prime}\right)\right)$ is equivalent to $\operatorname{sgn}\left(\tau-\tau^{\prime}\right)$. Keeping these in mind, one can find that the Wightman function along the uniformly accelerating detector takes the following form:

$$
\left\langle 0\left|\phi(x(\tau)) \phi\left(x\left(\tau^{\prime}\right)\right)\right| 0\right\rangle=\frac{\Gamma\left(\frac{d-2}{2}\right)}{4 \pi^{\frac{d}{2}}}\left[\frac{\pi T}{i \sinh \left(\pi T\left(\tau-\tau^{\prime}\right)\right)}\right]^{d-2} \text { for } \tau-\tau^{\prime} \neq 0,
$$

which satisfies the KMS condition $\langle 0|\phi(x(\tau)) \phi(x(0))| 0\rangle=\langle 0|\phi(x(0)) \phi(x(\tau+i / T))| 0\rangle$. As we will see shortly in the next section, the Fourier transform of (8) is exactly calculable. The result is that, when $d$ is even, $F(\omega)$ is proportional to the Bose-Einstein distribution and the proportional coefficient is a polynomial of $\omega$. This signifies that the uniformly accelerating detector registers the thermal spectrum of bosonic particles as if it were immersed in a heat bath, which would have been a major surprise in the early days of the field. But a real surprise is that, if $d$ is odd, $F(\omega)$ becomes proportional to the Fermi-Dirac distribution even in the absence of fermionic degrees of freedom. The resultant power spectrum turns out to be of the following forms:

$$
F(\omega)= \begin{cases}\frac{1}{\mathrm{e}^{\omega / T}-1} \times(\text { polynomial of } \omega) & \text { for } d \text { even } \\ \frac{1}{\mathrm{e}^{\omega / T}+1} \times(\text { polynomial of } \omega) & \text { for } d \text { odd }\end{cases}
$$

This is the Takagi's statistics inversion and one of the biggest bewildering results in the Unruh effect. And the reason why this happens can be explained-at least at the mathematical level—in terms of the Huygens principle $[8,10] .^{3}$ To see this, let us first note that the power spectrum (6) can be

\footnotetext{
${ }^{2}$ This is of course consistent with the $i \epsilon$ prescription: the Wightman function (7) is the boundary value (i.e., the limit $\epsilon \rightarrow+0)$ of the complex function $\langle 0|\phi(x) \phi(y)| 0\rangle \propto\left[-\left(x^{0}-y^{0}-i \epsilon\right)^{2}+|x-y|^{2}\right]^{-(d-2) / 2}=\left[(x-y)^{2}+i \epsilon \operatorname{sgn}\left(x^{0}-y^{0}\right)\right]^{-(d-2) / 2}$, which indicates that one has to approach the real $z$-axis from above (below) if $x^{0}-y^{0}>0\left(x^{0}-y^{0}<0\right)$. Notice that there is no discontinuity across the positive real z-axis.

${ }^{3}$ The Huygens principle roughly means that solutions of massless free field equations with sharply localized initial states propagate only along the future light-cone. In quantum field theory language, this is equivalent to the statement that the retarded Green's function $i \theta\left(x^{0}-y^{0}\right)\langle 0|[\phi(x), \phi(y)]| 0\rangle$ has a support only on the future light-cone $V_{+}=\left\{x^{\mu}-y^{\mu}\right.$ : $\left.(x-y)^{2}=0, x^{0}-y^{0} \geq 0\right\}$. This is true only for $d$ even. For $d$ odd, the retarded Green's function has a support on the closed future light-cone $\bar{V}_{+}=\left\{x^{\mu}-y^{\mu}:(x-y)^{2} \leq 0, x^{0}-y^{0} \geq 0\right\}$; that is, once one hears the sound of massless free fields, the reverberation never disappears in odd dimensional spacetime. This is the absence of Huygens principle, whose origin is the anti-commutation relation (11) for $d$ odd.
} 
recast into the following two equivalent forms: ${ }^{4}$

$$
\begin{aligned}
F(\omega) & =-\frac{1}{\mathrm{e}^{\omega / T}-1} \int_{-\infty}^{\infty} d \tau \mathrm{e}^{-i \omega \tau}\langle 0|[\phi(x(\tau)), \phi(x(0))]| 0\rangle \\
& =+\frac{1}{\mathrm{e}^{\omega / T}+1} \int_{-\infty}^{\infty} d \tau \mathrm{e}^{-i \omega \tau}\langle 0|\{\phi(x(\tau)), \phi(x(0))\}| 0\rangle,
\end{aligned}
$$

which just follow from the KMS condition. The second important point to note is that the Wightman function (7) for time-like separated operators satisfies the following exchange relation:

$$
\langle 0|\phi(x) \phi(y)| 0\rangle=\mathrm{e}^{-i \operatorname{sgn}\left(x^{0}-y^{0}\right)(d-2) \pi}\langle 0|\phi(y) \phi(x)| 0\rangle \text { for } \quad(x-y)^{2}<0,
$$

which becomes anti-commutation relation when $d$ is odd. Hence if $d$ is odd, the anti-commutator function $\langle 0|\{\phi(x(\tau)), \phi(x(0))\}| 0\rangle$ becomes zero for $\tau \neq 0$, which implies that it is non-vanishing only at $\tau=0$. Such function with a one-point support at $\tau=0$ must be given by a polynomial of the delta function $\delta(\tau)$ and its derivatives, whose Fourier transform (10b) must then become a polynomial of $\omega$. Similar arguments hold true for the commutator function $\langle 0|[\phi(x(\tau)), \phi(x(0))]| 0\rangle$ for $d$ even and its Fourier transform (10a). Putting all these things together, one arrives at the expressions (9). This is the Ooguri's explanation for the statistics inversion [10].

The Huygens principle offers a nice explanation for the form of detector's power spectrum (9), however, one may still find this hard to understand intuitively. Yet, regardless of the presence or absence of intuition, the computational origin of statistic inversion is obvious: it just comes from the power of the two-point Wightman function (7) (or (8)), which is just determined by the mass dimension of free scalar fields in $d$ dimensions. Hence it would be natural to expect that, if $\phi(x)$ had an anomalous dimension, the power spectrum could obey a thermal distribution function for some generalized statistics that interpolates the Bose-Einstein and Fermi-Dirac distributions continuously. As we shall see from now on, this is indeed the case if the detector's "monopole moment" couples to a scalar primary operator of conformal field theory with non-vanishing anomalous dimension.

\section{Statistics Interpolation: Emergent Anyon Distribution}

Let $\mathcal{O}_{\Delta}(x)$ be a hermitian scalar primary operator of scaling dimension $\Delta$ and couple to the "monopole moment" $m(\tau)$ via the interaction term $S_{\text {int }}=\int_{-\infty}^{\infty} d \tau m(\tau) \mathcal{O}_{\Delta}(x)$. At the linear order of perturbation theory, the detector's power spectrum is again given by (6) with $\phi$ being replaced by $\mathcal{O}_{\Delta}$. The two-point Wightman function of $\mathcal{O}_{\Delta}(x)$ is just determined from the conformal symmetry ${ }^{5}$ and takes the following forms:

$$
\left\langle 0\left|\mathcal{O}_{\Delta}(x) \mathcal{O}_{\Delta}(y)\right| 0\right\rangle= \begin{cases}\frac{\Gamma(2 \Delta) A_{\Delta}}{2 \pi} \frac{1}{\left[(x-y)^{2}\right]^{\Delta}} & \text { for } \quad(x-y)^{2}>0, \\ \frac{\Gamma(2 \Delta) A_{\Delta}}{2 \pi} \frac{\mathrm{e}^{-i \operatorname{sgn}\left(x^{0}-y^{0}\right) \pi \Delta}}{\left[-(x-y)^{2}\right]^{\Delta}} & \text { for } \quad(x-y)^{2}<0,\end{cases}
$$

where $A_{\Delta}$ is a dimensionless normalization factor which is assumed to be real. $\Gamma(2 \Delta) /(2 \pi)$ is factored out just for later convenience. It should be emphasized that, as noted in footnote 2 , the appearance of phase $\mathrm{e}^{-i \operatorname{sgn}\left(x^{0}-y^{0}\right) \pi \Delta}$ is consistent with the $i \epsilon$-prescription. One can easily check that eq. (12) enjoys the following properties:

\footnotetext{
${ }^{4}$ To derive these, use the translation invariance $\langle 0|\phi(x(0)) \phi(x(\tau))| 0\rangle=\langle 0|\phi(x(-\tau)) \phi(x(0))| 0\rangle$ and the KMS condition in frequency space, $F(-\omega)=\mathrm{e}^{\omega / T} F(\omega)$. For example, the Fourier transform of anti-commutator function is written as $\int_{-\infty}^{\infty} d \tau \mathrm{e}^{-i \omega \tau}\langle 0|[\phi(x(\tau)), \phi(x(0))]| 0\rangle=F(\omega)-F(-\omega)=\left(1-\mathrm{e}^{\omega / T}\right) F(\omega)$, which proves eq. (10a). Likewise, one can prove eq. (10b). It should be noted that eqs. (10a) and (10b) hold true for generic interacting fields with or without mass. The exchange relation (11), on the other hand, holds true only for massless free fields.

${ }^{5}$ For textbook exposition of $d$-dimensional conformal field theory, we refer to [14].
} 
- Hermiticity. The two-point Wightman function satisfies the identity

$$
\overline{\left\langle 0\left|\mathcal{O}_{\Delta}(x) \mathcal{O}_{\Delta}(y)\right| 0\right\rangle}=\left\langle 0\left|\mathcal{O}_{\Delta}(y) \mathcal{O}_{\Delta}(x)\right| 0\right\rangle \text { for } \forall x, y \in \mathbb{R}^{1, d-1},
$$

where the overline stands for the complex conjugate. This is just a manifestation of hermiticity $\mathcal{O}_{\Delta}^{\dagger}(x)=\mathcal{O}_{\Delta}(x)$ for the scalar primary operator.

- Space-like commutativity. The two-point Wightman functions for space-like separated operators commute with each other:

$$
\left\langle 0\left|\mathcal{O}_{\Delta}(x) \mathcal{O}_{\Delta}(y)\right| 0\right\rangle=\left\langle 0\left|\mathcal{O}_{\Delta}(y) \mathcal{O}_{\Delta}(x)\right| 0\right\rangle \text { for }(x-y)^{2}>0 .
$$

This is just a manifestation of causality. ${ }^{6}$

- Discontinuity across the branch cut. The two-point Wightman function for time-like separated operators acquires a phase when $\mathcal{O}_{\Delta}(x)$ and $\mathcal{O}_{\Delta}(y)$ are swapped with each other:

$$
\left\langle 0\left|\mathcal{O}_{\Delta}(x) \mathcal{O}_{\Delta}(y)\right| 0\right\rangle=\mathrm{e}^{-i \operatorname{sgn}\left(x^{0}-y^{0}\right) 2 \pi \Delta}\left\langle 0\left|\mathcal{O}_{\Delta}(y) \mathcal{O}_{\Delta}(x)\right| 0\right\rangle \text { for } \quad(x-y)^{2}<0 .
$$

This is just a manifestation of multi-valuedness as a function of $z=(x-y)^{2}$ and resulting discontinuity across the branch cut along the negative $z$-axis.

Notice that the appearance of phase does not conflict with the hermiticity (13).

Now, having the Wightman function for time-like separated operators, one can easily find the Wightman function along the detector's worldline (3) by just replacing $-(x-y)^{2}$ with $-(x(\tau)-$ $\left.x\left(\tau^{\prime}\right)\right)^{2}=\left|\sinh \left(\pi T\left(\tau-\tau^{\prime}\right)\right)\right|^{2} /(\pi T)^{2}$. For $\tau \neq \tau^{\prime}$, the result is

$$
\begin{aligned}
\left\langle 0\left|\mathcal{O}_{\Delta}(x(\tau)) \mathcal{O}_{\Delta}\left(x\left(\tau^{\prime}\right)\right)\right| 0\right\rangle & =\frac{\Gamma(2 \Delta) A_{\Delta}}{2 \pi} \mathrm{e}^{-i \operatorname{sgn}\left(\tau-\tau^{\prime}\right) \pi \Delta}\left[\frac{\pi T}{\left|\sinh \left(\pi T\left(\tau-\tau^{\prime}\right)\right)\right|}\right]^{2 \Delta} \\
& =\frac{\Gamma(2 \Delta) A_{\Delta}}{2 \pi}\left[\frac{\pi T}{i \sinh \left(\pi T\left(\tau-\tau^{\prime}\right)\right)}\right]^{2 \Delta},
\end{aligned}
$$

which, up to the overall normalization factor, exactly coincides with the chiral half of the two-point Wightman function for $(1+1)$-dimensional anyon field at finite temperature $T=a /(2 \pi)[12,16-20]$, where the proper time $\tau$ plays the role of the light-cone coordinates $x^{ \pm}=x^{0} \pm x^{1}$ of two-dimensional Minkowski spacetime. In fact, eq. (16) has the desired properties for anyon two-point Wightman functions at finite temperature:

- Hermiticity.

$$
\overline{\left\langle 0\left|\mathcal{O}_{\Delta}(x(\tau)) \mathcal{O}_{\Delta}\left(x\left(\tau^{\prime}\right)\right)\right| 0\right\rangle}=\left\langle 0\left|\mathcal{O}_{\Delta}\left(x\left(\tau^{\prime}\right)\right) \mathcal{O}_{\Delta}(x(\tau))\right| 0\right\rangle \quad \text { for } \quad \forall \tau, \tau^{\prime} \in \mathbb{R} .
$$

\section{- Anyon exchange relation.}

$$
\left\langle 0\left|\mathcal{O}_{\Delta}(x(\tau)) \mathcal{O}_{\Delta}\left(x\left(\tau^{\prime}\right)\right)\right| 0\right\rangle=\mathrm{e}^{-i \operatorname{sgn}\left(\tau-\tau^{\prime}\right) 2 \pi \Delta}\left\langle 0\left|\mathcal{O}_{\Delta}\left(x\left(\tau^{\prime}\right)\right) \mathcal{O}_{\Delta}(x(\tau))\right| 0\right\rangle \text { for } \quad \tau \neq \tau^{\prime} .
$$

\section{- KMS condition.}

$$
\left\langle 0\left|\mathcal{O}_{\Delta}(x(\tau)) \mathcal{O}_{\Delta}\left(x\left(\tau^{\prime}\right)\right)\right| 0\right\rangle=\left\langle 0\left|\mathcal{O}_{\Delta}\left(x\left(\tau^{\prime}\right)\right) \mathcal{O}_{\Delta}\left(x\left(\tau+\frac{i}{T}\right)\right)\right| 0\right\rangle \quad \text { for } \quad \forall \tau, \tau^{\prime} \in \mathbb{R} .
$$

\footnotetext{
${ }^{6}$ Strictly speaking, causality is an intricate problem in conformal field theory because finite special conformal transformations generally change the sign of the Poincaré invariant $(x-y)^{2}$. (The maximal symmetry transformations that preserve the causal structure of Minkowski spacetime are only the Poincaré transformations and dilatation.) In order to make the special conformal transformations both globally well-defined and causal, one needs to work in the universal covering of conformally compactified Minkowski spacetime; see, e.g., ref. [15] for a short review. In this note we will not touch upon this causality issue.
} 
Notice that the scaling dimension $\Delta$ plays the role of the so-called statistical parameter $\kappa=2 \Delta$, which interpolates the Bose-Einstein and Fermi-Dirac statistics in a continuous way. Indeed, the power spectrum $F(\omega)$ interpolates the Bose-Einstein and Fermi-Dirac distributions. To see this, we need to Fourier-transform the Wightman function (16), which can be done as follows:

$$
\begin{aligned}
F(\omega) & =\int_{-\infty}^{\infty} d \tau \mathrm{e}^{-i \omega \tau}\left\langle 0\left|\mathcal{O}_{\Delta}(x(\tau)) \mathcal{O}_{\Delta}(x(0))\right| 0\right\rangle \\
& =\frac{\Gamma(2 \Delta) A_{\Delta}}{2 \pi} \int_{-\infty}^{\infty} d \tau \mathrm{e}^{-i \omega \tau}\left[\frac{\pi T}{i \sinh (\pi T(\tau-i \epsilon))}\right]^{2 \Delta} \\
& =\frac{\Gamma(2 \Delta) A_{\Delta}}{2 \pi} \int_{-\infty-\frac{i}{2 T}}^{\infty} d \tau \mathrm{e}^{-i \omega \tau}\left[\frac{\pi T}{i \sinh (\pi T \tau)}\right]^{2 \Delta} \\
& =\frac{\Gamma(2 \Delta) A_{\Delta}}{2 \pi}(2 \pi T)^{2 \Delta-1} \mathrm{e}^{-\frac{\omega}{2 T}} \int_{0}^{\infty} d z \frac{z^{\Delta-\frac{i \omega}{2 \pi T}-1}}{(1+z)^{2 \Delta}} \\
& =\frac{A_{\Delta}}{2 \pi}(2 \pi T)^{2 \Delta-1} \mathrm{e}^{-\frac{\omega}{2 T}}\left|\Gamma\left(\Delta+\frac{i \omega}{2 \pi T}\right)\right|^{2},
\end{aligned}
$$

where in the third equality we have pushed the integration contour slightly downward by $i /(2 T)$ in such a way that the integrand does not blow up along the contour. The fourth equality follows from the change of variable $z=\mathrm{e}^{2 \pi T(\tau+i /(2 T))}$ and the last equality follows from the integral expression for the beta function:

$$
B(p, q)=\int_{0}^{\infty} d z \frac{z^{p-1}}{(1+z)^{p+q}}=\frac{\Gamma(p) \Gamma(q)}{\Gamma(p+q)} \quad \text { for } \quad \operatorname{Re} p>0 \& \operatorname{Re} q>0
$$

where $p=\Delta-i \omega /(2 \pi T)$ and $q=\Delta+i \omega /(2 \pi T)$. Notice that $|\Gamma(x+i y)|^{2}=\Gamma(x+i y) \Gamma(x-i y)$ for $x, y \in \mathbb{R}$. Note also that the power spectrum (20) satisfies the KMS condition in frequency space:

$$
F(-\omega)=\mathrm{e}^{\omega / T} F(\omega) .
$$

In order to see the statistics interpolation, we first note that, just as in eqs. (10a) and (10b), the power spectrum (20) can be recast into the following suggestive forms for any $\Delta$ :

$$
F(\omega)=A_{\Delta} \frac{(2 \pi T)^{2 \Delta}}{\omega}\left|\frac{\Gamma\left(\Delta+\frac{i \omega}{2 \pi T}\right)}{\Gamma\left(\frac{i \omega}{2 \pi T}\right)}\right|^{2} \frac{1}{\mathrm{e}^{\omega / T}-1}=A_{\Delta}(2 \pi T)^{2 \Delta-1}\left|\frac{\Gamma\left(\Delta+\frac{i \omega}{2 \pi T}\right)}{\Gamma\left(\frac{1}{2}+\frac{i \omega}{2 \pi T}\right)}\right|^{2} \frac{1}{\mathrm{e}^{\omega / T}+1},
$$

which follows from the Euler's reflection formulae $|\Gamma(i x)|^{2}=\pi /(x \sinh (\pi x))$ and $|\Gamma(1 / 2+i x)|^{2}=$ $\pi / \cosh (\pi x)$ for $x \in \mathbb{R}$. Now it is easy to see that, when $\Delta$ is either an integer or a half-odd integer, the Gamma function factors reduce to polynomials of $\omega$. In these cases the power spectrum results in the following:

$$
F(\omega)=\left\{\begin{array}{l}
\frac{A_{\Delta} \omega^{2 \Delta-1}}{\mathrm{e}^{\omega / T}-1} \prod_{n=1}^{\Delta-1}\left[1+\left(\frac{2 n \pi T}{\omega}\right)^{2}\right] \text { for } \quad \Delta=1,2, \cdots, \\
\frac{A_{\Delta} \omega^{2 \Delta-1}}{\mathrm{e}^{\omega / T}+1} \prod_{n=\frac{1}{2}}^{\Delta-1}\left[1+\left(\frac{2 n \pi T}{\omega}\right)^{2}\right] \text { for } \quad \Delta=\frac{1}{2}, \frac{3}{2}, \cdots,
\end{array}\right.
$$

where for $\Delta=1$ and $1 / 2$ the products should be disregarded. These give the explicit expressions of the power spectrum (9) for massless free scalar fields if the scaling dimension is $\Delta=(d-2) / 2$, $d \in\{3,4,5, \cdots\}$. Hence for generic $\Delta$, the power spectrum (20) interpolates the Bose-Einstein and 


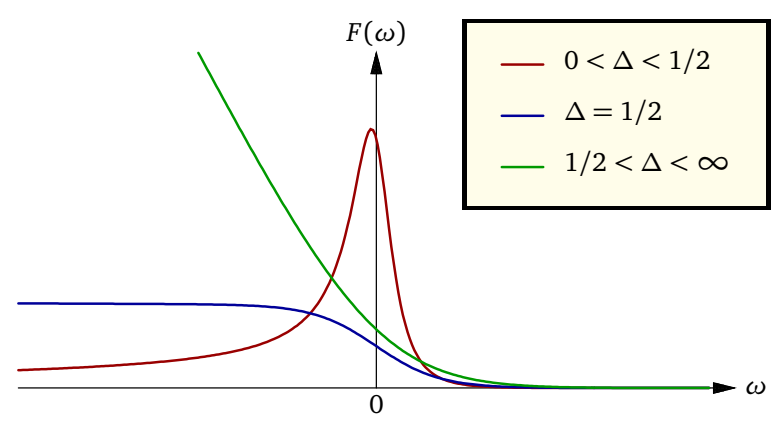

Figure 1: Typical detector's power spectra. The case $\omega=E_{f}-E_{i}>0$ corresponds to the absorption process while the case $\omega=E_{f}-E_{i}<0$ corresponds to the emission process. Note that the scaling dimension $\Delta$ is bounded below by $(d-2) / 2$ in unitary conformal field theories.

Fermi-Dirac distributions continuously. In fact, it coincides with the so-called momentum distribution of $(1+1)$-dimensional chiral anyons at finite temperature $[12,20]$. Typical power spectra are depicted in figure 1.

To summarize, we have seen that, as a consequence of the branch point singularity, the conformal two-point function generally obeys the anyon(-like) exchange relation in the time-like domain. Its Fourier transform along the worldline of uniformly accelerating detector then coincides with the thermal distribution for $(1+1)$-dimensional anyons at the Unruh temperature $T=a /(2 \pi)$. It is worth mentioning here that the detector's power spectrum enjoys yet another geometrical interpretation. As briefly noted in refs. $[8,10]$, the Fourier transform $F(\omega)$ is related to the nontrivial density of states on the Rindler wedge. In fact, for the case of canonical dimension $\Delta=(d-2) / 2$, the power spectrum is proportional to the Plancherel measure for a scalar field on the $(d-1)$-dimensional hyperbolic space $\mathbb{H}^{d-1}$ (see, e.g., eq. (2.36) in ref. [21]): ${ }^{7}$

$$
\mu_{0}(\lambda) \propto\left|\frac{\Gamma((d-2) / 2+i \lambda)}{\Gamma(i \lambda)}\right|^{2},
$$

where $\lambda$ should be read as $\omega /(2 \pi T)$. Obviously, eq. (25) can be analytically continued to arbitrary $\Delta=(d-2) / 2$. Hence the detector's power spectrum (23) allows two distinct interpretations: one is the Bose-Einstein distribution multiplied by the Plancherel measure, and the other is the anyon distribution. The Takagi's statistics inversion thus comes from the peculiar property of this Plancherel measure. It is quite interesting that the Unruh effect gives a geometrical interpretation for the anyon distribution and may well provide a surprising connection between statistics and geometry.

\section{Acknowledgments}

The author would like to thank Mihail Mintchev for discussions and comments. He is also grateful to INFN Pisa for hospitality during his visit at the University of Pisa. This work was supported in part by JSPS Grant-in-Aid for Research Activity Startup \#15H06641.

\section{References}

[1] W. G. Unruh, "Notes on black-hole evaporation," Phys. Rev. D14 (1976) 870-892.

[2] S. W. Hawking, "Particle Creation by Black Holes," Commun. Math. Phys. 43 (1975) 199-220.

[3] Y. Takahashi and H. Umezawa, "Thermo field dynamics," Int. J. Mod. Phys. B10 (1996) 1755-1805.

\footnotetext{
${ }^{7}$ Notice that the $d$-dimensional Rindler wedge is conformal to $\mathbb{H}^{1} \times \mathbb{H}^{d-1}$; see, e.g., ref. [22].
} 
[4] J. J. Bisognano and E. H. Wichmann, "On the duality condition for a Hermitian scalar field," J. Math. Phys. 16 (1975) 985-1007.

[5] J. J. Bisognano and E. H. Wichmann, "On the duality condition for quantum fields," J. Math. Phys. 17 (1976) 303-321.

[6] R. Haag, N. M. Hugenholtz, and M. Winnink, "On the equilibrium states in quantum statistical mechanics," Commun. Math. Phys. 5 (1967) 215-236.

[7] G. L. Sewell, "Quantum fields on manifolds: PCT and gravitationally induced thermal states," Annals Phys. 141 (1982) 201-224.

[8] S. Takagi, "Vacuum noise and stress induced by uniform accelerator: Hawking-Unruh effect in Rindler manifold of arbitrary dimensions," Prog. Theor. Phys. Suppl. 88 (1986) 1-142.

[9] B. S. DeWitt, "Quantum gravity: the new systhesis," in General Relativity: An Einstein Centenary Survey, S. W. Hawking and W. Israel, eds., pp. 680-745. Cambridge University Press, Cambridge, 1979.

[10] H. Ooguri, "Spectrum of Hawking radiation and the Huygens principle," Phys. Rev. D33 (1986) 3573-3580.

[11] R. Courant and D. Hilbert, Methods of Mathematical Physics, Volume II: Partial Differential Equations. Interscience Publishers, New York, 1962.

[12] A. Liguori, M. Mintchev, and L. Pilo, "Bosonization at finite temperature and anyon condensation," Nucl. Phys. B569 (2000) 577-605, arXiv: hep-th/9906205 [hep-th] .

[13] L. Sriramkumar, "Interpolating Between the Bose-Einstein and the Fermi-Dirac Distributions in Odd Dimensions," Gen. Rel. Grav. 35 (2003) 1699-1705, arXiv:gr-qc/0212084 [gr-qc].

[14] E. S. Fradkin and M. Y. Palchik, Conformal Quantum Field Theory in D-dimensions, vol. 376 of Mathematics and Its Applications. Kluwer, Dordrecht, 1996.

[15] H. A. Kastrup, "On the Problem of Causality in Conformal Invariant Theories," in Theories and Experiments in High-Energy Physics, A. Perlmutter and S. M. Widmayer, eds., pp. 411-418. Plenum Press, New York, 1975.

[16] N. Ilieva and W. E. Thirring, "Anyons and the Bose-Fermi duality in the finite temperature Thirring model," Theor. Math. Phys. 121 (1999) 1294-1314, arXiv:math-ph/9906020 [math-ph]. [Erratum: Theor. Math. Phys. 125 (2000) 1742].

[17] N. Ilieva, H. Narnhofer, and W. Thirring, "Thermal correlators of anyons in two dimensions," J. Phys. A34 (2001) 3083-3094, arXiv:math-ph/0004006 [math-ph].

[18] N. Ilieva and W. E. Thirring, "Laughlin type wave function for two-dimensional anyon fields in a KMS-state," Phys. Lett. B504 (2001) 201-206, arXiv:hep-th/0010030 [hep-th].

[19] N. Ilieva, "Two-dimensional anyons and the temperature dependence of commutator anomalies," Int. J. Mod. Phys. A16 (2001) 1407-1416, arXiv:hep-th/0101140 [hep-th].

[20] M. Mintchev and P. Sorba, "Luttinger liquid in a non-equilibrium steady state," J. Phys. A46 (2013) 095006, arXiv:1210.5409 [math-ph].

[21] R. Camporesi and A. Higuchi, "Spectral functions and zeta functions in hyperbolic spaces," J. Math. Phys. 35 (1994) 4217-4246. 
[22] S. Ohya, "Intertwining operator in thermal CFT ${ }_{d}$," Int. J. Mod. Phys. A32 (2017) 1750006, arXiv: 1611.00763 [hep-th]. 